Culture et histoire dans l'espace roman

4 | 2010

Les représentations du corps dans la litterature latinoaméricaine

\title{
Las aventuras del Sr. Maíz, de Cucurto : du corps et du capitalisme
}

\section{Geneviève Orssaud}

\section{OpenEdition}

\section{Journals}

Édition électronique

URL : https://journals.openedition.org/cher/8379

DOI : $10.4000 /$ cher.8379

ISSN : 2803-5992

Éditeur

Presses universitaires de Strasbourg

Édition imprimée

Date de publication : 30 juin 2010

Pagination : 159-171

ISBN : 978-2-35410-007-0

ISSN : 1968-035X

Référence électronique

Geneviève Orssaud, "Las aventuras del Sr. Maíz, de Cucurto : du corps et du capitalisme », reCHERches [En ligne], 4 | 2010, mis en ligne le 15 décembre 2021, consulté le 26 janvier 2022. URL : http:// journals.openedition.org/cher/8379; DOI : https://doi.org/10.4000/cher.8379

\section{(c) (i) (8)}

Ce(tte) œuvre est mise à disposition selon les termes de la Licence Creative Commons Attribution -

Pas d'Utilisation Commerciale - Partage dans les Mêmes Conditions 4.0 International. 


\title{
Las aventuras del Sr. Maíz, de Cucurto: du corps et du capitalisme
}

\author{
GeNEVIÈVE ORSSAUD \\ Université Paris III - Sorbonne Nouvelle
}

\begin{abstract}
Cucurto' ${ }^{1}$, poète et romancier, est né en 1973 en Argentine. Son actualité est faite du néolibéralisme et du chaos politique qui ont suivi les périodes des dictatures. Il évoque dans ses textes une Argentine métisse, située en Amérique du Sud. Il se distingue par là du courant intellectuel de la littérature argentine, qui établit plus de liens avec les écoles littéraires européennes qu'avec celles de ses pays voisins.

Il publie son roman Las aventuras del Sr. Maíz en 2005. Le titre est en deux parties, mais on ne découvre la seconde qu'en page de titre: El héroe atrapado entre dos mundos. Le protagoniste, dénommé Cucurto, est un poète autodidacte, magasinier dans un supermarché, danseur invétéré de cumbia et amant dédié aux immigrées dominicaines. Il est poussé par une de ses amantes à se faire recouvrir le sexe d'or afin d'être élu Señor Maíz, un saint dominicain qui pourrait sauver la République Dominicaine de la pauvreté et de la famine. La narration prend place dans une représentation très réaliste des quartiers pauvres du centre de Buenos Aires. Ce roman est l'occasion pour l'auteur de présenter une lecture sociale de l'Argentine contemporaine.
\end{abstract}

1 Les premiers textes de Cucurto sont signés Washington Cucurto, mais à partir de Las aventuras del Sr. Maíz, le prénom disparaît. C'est pourquoi je ne le fais pas apparaître ici. 
L'auteur et le personnage portent le même nom. En réalité, l'auteur est né sous le nom de Santiago Vega, mais signe ses textes Cucurto et répond à ce nom dans les entretiens. L'origine de ce nom est narrée dans plusieurs de ses textes, de façon sensiblement différente. Ce qu'on peut en retenir c'est que ce pseudonyme lui a été donné par d'autres poètes, lors de ses premiers contacts avec le monde littéraire portègne ${ }^{2}$. Le fait que ce "baptême» apparaisse dans ses fictions et soit le fait de poètes en fait un nom littéraire. Auteur et personnage prennent le même nom et commencent à exister en tant que tels dans le même temps - ils sont liés par leurs conditions littéraires: l'auteur crée son personnage, et en tant que personnage, ce dernier fait de son créateur un auteur littéraire. C'est ce qui, à mon sens, nous écarte de l'autofiction. Le personnage créé par Cucurto est présent dans plusieurs de ses textes, est poète comme lui, et est inspiré d'expériences vécues par l'auteur, mais il est plus une extrapolation qu'une représentation de l'auteur. La fiction est une possibilité où le personnage peut prendre n'importe quelle forme, un lieu d'expérimentation pour Cucurto comme pour n'importe quel auteur avec n'importe lequel de ses personnages. Dans l'univers de Cucurto, le personnage est avant tout un média, un passeur entre réel et fiction. Il s'adresse constamment au lecteur, faisant de ce dernier un personnage de plus dans la narration, parfois amené à intervenir dans le récit. C'est un lecteur stéréotypé, qui représente le regard de la classe moyenne. C'est lui que le personnage cherche à introduire dans la représentation de quartiers peu connus mais bien réels de la capitale afin qu'il se trouve au contact d'une population marginale, faite des diverses immigrations sud-américaines et caribéennes. Ces quartiers et leurs habitants entrent ainsi dans le réel du lecteur après qu'il en a pris connaissance dans le roman. Mais les adresses du narrateur au lecteur sont omniprésentes dans le texte, également loin des passages où le lecteur apparaît en tant que personnage. L'auteur entraîne dans la fiction tous ses lecteurs, grâce à des points de correspondance entre le réel et la fiction: son nom fictif dans le réel et un fort réalisme dans la fiction. En effet, Las aventuras del Sr. Maíz est très réaliste, bien que le réel y soit représenté de façon exubérante. C'est ce que l'auteur appelle le "realismo atolondrado", tracé à grands traits, ainsi assimilable par le lecteur plus facilement et sans réticences. Passages entre fiction et réel, donc, facilités par un auteur et un personnage qui partagent leur nom.

Pour faciliter la lecture de ce travail, j'appellerai le personnage par son nom et je préciserai quand il s'agira de l'auteur.

2 De Buenos Aires. 
Las aventuras del Sr. Maíz commence avec la naissance du personnage Cucurto: "Llego al mundo. Peso 2,30 kg», sont les deux premières phrases $\mathrm{du}$ roman. Cette ouverture, qui narre l'entrée physique du personnage dans le monde, favorise l'immersion fictionnelle du lecteur en marquant un passage vers le monde fictionnel. Cette naissance dans un texte de fiction, c'est-à-dire dans un univers où la chair n'existe pas, indique toute l'importance du corps dans ce roman. On pourrait dire, dans la mesure où ce sont les premières lignes du texte, que ce personnage est corps. La narration est située en 1990, dans le contexte du gouvernement du président néolibéraliste Carlos Saúl Menem. L'emploi de magasinier de Cucurto n'est donc pas anodin. "Yo repuse para el neoliberalismo argentino, década del 90, en Carrefour [...] yo viví, cogí, cumbiantié, reponí, comí, para el neoliberalismo» (Cucurto 2005: 71). Les activités physiques essentielles sont ici liées au travail et à une époque qui donne au travail une valeur marchande. La première condition de Cucurto est alors celle de corps ouvrier. Il se trouve dans un monde de consumérisme exacerbé et il travaille pour une chaîne internationale de supermarchés, c'est-à-dire au cœur d'un haut lieu du capitalisme, qui en devient le symbole dans le cadre de la narration:

Alarmas en el piso, en el techo, en el cielo, en el sol, en el culo de las cajeras, en el pelo de los niños. Para no alarmarse, con tantas alarmas. Sonrían papas, perejiles, apios, verdeos, pomelos, ajos, balanzas, repositores, quiero a todo el mundo sonriendo, sonrisas de oreja a culo, sonrían, sonrían iy sonrían! (Cucurto 2005: 29)

Cucurto présente ce lieu comme un système clos, qui prévoit de punir des clients si violemment attirés qu'ils sont considérés comme des voleurs potentiels. L'employé, aussi accessoire que les produits présentés, est un rouage de cette machine qui ne peut faillir. Le travailleur est en effet une pièce centrale du monde capitaliste, ce qui le rend à la fois indispensable et absolument remplaçable. Le corps ouvrier est déformé:

Regalamos horas, muchos nos jodimos la cintura, la espalda, nos agarraban alergias del frío de las cámaras, dolor de huesos en pies y tobillos. Cortes de dedos, quebraduras de brazos y encima trabajando gratis. ¿Para quién nos estábamos sacrificando [...]? Para un señor y sus amigotes. (Cucurto 2005: 54)

Le corps est ce qui se monnaie dans le monde capitaliste, le premier produit échangeable. C'est ce dont profite le "señor", à la tête de ce système libéraliste, mais aussi le travailleur quand il dépense son salaire. Comme l'ajoute le narrateur: «Yo con mi recibo podía coger 85 mulatas dominicanas exactas!» (Cucurto 2005: 54) 
Or, tous les corps ouvriers que nous lisons dans le roman sont des "Noirs», c'est-à-dire selon l'acception la plus répandue en Amérique $\mathrm{du}$ Sud, les indiens, qu'ils soient norteños (des provinces du nord de l'Argentine), Péruviens, ou Dominicains; mais ce sont aussi ceux qui «trabajan como negros», c'est-à-dire comme des esclaves, selon l'expression utilisée en Argentine. Ils sont cette main-d'œuvre remplaçable qui permet au capitalisme d'exister: "chau, se va otro negro, otro negro desaparece» (Cucurto 2005: 74). Dans le monde décrit par l'auteur, les Noirs travaillent et les Blancs ${ }^{3}$ profitent. Ces derniers se font l'écho de ceux venus il y a cinq siècles, les conquistadores, dévorateurs de culture:

por qué chucha creen que hablamos español y los brasileños portugués. Shoppings, edificios, empresas, aviones, cumbia, putas, de dónde salieron, quién los inventó y peor aun, qué diablo los puso en nuestras vidas. [...] Alguien, una bomba, un planeta, que acabe con esta pinche raza putrefacta (Cucurto 2005: 80-81)

Le monde néolibéraliste décrit par Cucurto est le fait de ces envahisseurs, qui se sont approprié le continent américain et ses richesses. Cette «race», ce n'est pas la race blanche mais la "race capitaliste»: " "Servirlo", es la palabra que más le gusta a esta parche y pinche clase clienteril de ricos y pobres, putos y lesbianas, niños y jubilados, negros y blancos, yanquis o árabes" (Cucurto 2005: 34-35).

Cucurto, qui dit de lui-même "Yo era un negro» (Cucurto 2005: 48), fréquente le monde des Noirs. C'est un monde de travailleurs immigrés d'autres pays du continent comme d'autres provinces du pays. Ils sont des ouvriers Noirs, c'est-à-dire les plus pauvres. Ils sont attirés par la capitale argentine précisément parce que, en plein néolibéralisme, elle vit au rythme du Primer Mundo ${ }^{4}$. Cucurto insiste avec ironie sur le rejet dont ils sont l'objet, de la part des «Blancs»: «La Raza Inferior Multinforme, futbolera, machista y retrógrada fluyendo por todas partes. Bueno, a rodearme y embadurnarme con la mugre de la turba horrible, que nace de la mezcla del indio con el español» (Cucurto 2005: 61). Il fait des termes de ce mépris de hautes qualifications - ce qu'indiquent les majuscules en début de citation - les vidant ainsi de leur sens. Mais quoiqu'il en dise, ils pénètrent ainsi en masse

3 Je dois préciser ici que le terme «blanco» n'apparaît pas dans Las aventuras, ni les «Blancs» eux-mêmes. Ils sont en marge du texte, leur présence est suggérée comme une menace omniprésente. J'utilise donc le terme «Blanc» en opposition aux negros du roman, qui s'opposent en réalité eux-mêmes aux Blancs. En effet, l'auteur utilise ce terme sans faire de distinction entre les personnes d'origine indienne (les negros argentins) ou africaine (les negros dominicains), ou encore les travailleurs.

4 Terme dérivé de «Tiers-monde», utilisé pour désigner les pays riches. 
dans le roman. Le narrateur les définit d'une façon exubérante qui les fait exister. Ces immigrés ont le secret de la vie: «me enseñaron a ver la vida de otra manera, me enseñaron que en la tristeza y la miseria se baila igual y que hagamos lo que hagamos jamás mejoraremos en nada» (Cucurto 2005: 55). Et ils sont effectivement, dans ce roman, une foule dansante, riante, et aimante. Ils célèbrent la vie chaque jour, bien qu'elle soit si dure pour eux. L'auteur montre ainsi la richesse des populations immigrées à Buenos Aires, une capitale pourtant habituellement considérée comme une ville européenne, par les pays voisins comme par les portègnes eux-mêmes. Mais précisément, c'est l'Amérique et ses populations mêlées qui pénètrent le cœur du néolibéralisme, associé dans ce roman au néocolonialisme ${ }^{5}$, à travers ces prostituées métisses et mulâtresses qui entrent à flots à Buenos Aires. Cette richesse est invisible, il faut savoir et surtout vouloir la voir: «Tales cosas se daban en aquel yoti invisible para el resto de los habitantes de la ciudad y conocidísimo sólo para algunos maleantes o locos incurables como yo" (Cucurto 2005: 63). Si elle est invisible c'est parce qu'elle s'oppose radicalement à la richesse capitaliste. Elle s'y oppose en premier lieu par l'allégresse de ces immigrés: ce sont des pauvres heureux, ce qui va contre tous les préceptes capitalistes. Ensuite parce que leur échelle de valeurs est incompatible avec celle du profit. Comme nous le dit Cucurto, pour eux une chaîne en or impose plus de respect que la puissance: «Si una noche de otoño vas por Constitución con una cadenita en el cuello, serás una especie de Hijo del Presidente del Paraguay. Serás vos el Rey de la Cumbia y no los Sellos Discográficos Piratas como Líder Music» (Cucurto 2005 : 14).

L'auteur réintroduit par le biais de la fiction le tropicalisme à Buenos Aires aujourd'hui, qui comme tous les ports espagnols à l'époque de la colonisation était une sorte de seuil entre l'Europe et l'Amérique. Les Noirs sont présentés comme des sauvages, des êtres de la jungle, à l'image desquels doit être le sexe du prétendant au titre de Sr. Maíz: «Le puse la pija encima de la camilla, dormida, vivaracha, expectante al primer roce pa despertarse como un animal salvaje. Tropical» (Cucurto 2005: 18). Ce tropicalisme n'est pas celui des populations barbares que les Espagnols découvrent, mais la résultante de divers métissages, le peuple américain actuel, ni européen, ni indien. Ce métissage est l'identité américaine, et donc l'identité argentine.

5 Terme utilisé par Luis Felipe Noé pour désigner les nouvelles formes de la colonisation, notamment économique et culturelle (Noé 2003). 
Par le biais de l'opposition entre les Noirs et les Blancs - je le redis, repoussés en dehors du texte - l'auteur revendique l'identité de son pays ${ }^{6}$.

Outre la fonction ouvrière du corps, la jouissance est amplement explorée dans le roman, notamment par le narrateur lui-même. Cucurto aime le sexe, et jouit de plaisirs extrêmes. Il aime les prostituées, les très jeunes filles et les femmes âgées, qui lui rappellent sa mère. Il apprécie aussi les rapports avec un animal. Plus que d'un pervers il s'agit d'un amant universel, ou plutôt d'un corps, et d'un phallus en particulier, qui est accessible à tous, du fait de sa perfection. Son phallus provoque l'adoration: «Le dio un beso a la punta de mi poronga como todos los días. Se persignó ante el Dios Morcillón» (Cucurto 2005: 16). C’est ce sexe si parfait «como Dios, como la creación de la vida» (Cucurto 2005: 18), qui fait de lui le Sr. Maíz, un corps sacré qui appartient à tous, à tel point qu'il peut être violé sans offense. En sa qualité d'être divin, il est possédé par tous. Ce personnage fait référence à un dieu de la mythologie dominicaine, reconnaissable à son phallus d'or, qui, à son arrivée sur terre, garantira la prospérité de la République Dominicaine en la protégeant de la famine. Il est représenté par l'auteur à la façon d'un saint populaire, comme on en trouve beaucoup en Amérique latine. On peut reconnaître en lui d'autres dieux aux mêmes attributs, tels que le dieu gallo-romain Priape, un dieu d'ailleurs proprement populaire, mais aussi Quetzalcóatl, dans certaines de ses interprétations - je pense à la description qu'en fait Carlos Fuentes dans Todos los gatos son pardos: «El AUGUR/ QUETZALCÓATL se levanta la túnica y muestra, entre las piernas y detenido con ambas manos, un gigantesco falo de oro" (Fuentes 1990: 28). On reconnaît chez le Sr. Maíz deux traits proprement américains: le maïs, dont la culture est la première richesse des civilisations précolombiennes, et vénéré comme un don du ciel, et l'or, tant convoité par les conquistadores, un métal dont on dit par ailleurs qu'il brille comme le soleil et dont l'inaltérabilité en a fait le symbole du divin. Ce sexe d'or est donc un attribut du pouvoir, comme c'est d'ailleurs très clairement écrit: «- $-\dot{L}$ a ven? Es de Oro, pesa dos kilos y acá mando yo» (Cucurto 2005: 85). Dans cette

6 Je voudrais signaler ici que ce discours identitaire en fait l'héritier de la littérature argentine des années 1960, qui a cherché à définir l'identité argentine, en incluant à la fois la "pré-histoire» des Argentins (leur passé indien, avant l'écriture de l'histoire et la constitution de la nation argentine) et leur ralliement à l'histoire de l'Europe, à partir de la «découverte». Je donne pour référence ce précédent travail, "Échos de la quête de l'identité argentine au $\mathrm{XX}^{\mathrm{e}}$ siècle dans un récit fictionnel de la colonisation: Zama d'Antonio Di Benedetto", in Trans-, $\mathrm{n}^{\circ}$ 5, janvier 2008. Source: http://trans.univ-paris3. fr/spip.php?article212 
démonstration un peu ridicule du pouvoir point évidemment la critique. Ce faux saint, "imposteur» comme se dit le personnage, qui compare la taille de son sexe avec ses camarades est l'occasion pour l'auteur de critiquer le pouvoir capitaliste, un système dans lequel celui qui s'impose le plus est le plus respecté. Il le reconnaît dans un entretien:

Eso es una leyenda dominicana muy común: creen que va a venir un dios con el falo de oro, pero como todavía no viene, lo reemplazan por un dios terrenal que es el que la tiene más grande. Es como una parodia del capitalismo, ¿no? Allá es algo religioso, pero acá se puede leer como una crítica a la sociedad. (Nicolini 2006)

Cucurto est adoré pour les proportions de son corps, pas pour sa spiritualité. Quant à lui, s'il accepte de jouer au saint, ce qu'il ne fera pas plus de vingt-quatre heures, c'est pour profiter de l'adoration que lui vouent les Dominicaines. La consécration du Sr. Maíz donne lieu à des scènes burlesques: «Y yo [...] sacudía [el pene], se los enseñaba rodeado de flores, crucifijos, rosarios, remeras, fotitos de personas enfermas, banderas y casacas de los equipos de Béisbol» (Cucurto 2005: 79). On reconnaît ici la profusion de démonstrations qui font le culte religieux tel qu'il est pratiqué en Amérique du Sud, qui tient plus de la superstition que de la foi. Le contraste entre ce phallus et les codes de la religion catholique provoque ce qui semble être une croyance totalement absurde, ce que l'auteur souligne par le biais de l'humour, comme dans le passage où la bijoutière découvre le sexe de Cucurto: «puse mis dos huevos negros sobre el blanco ala reluciente de la camilla.

- Ay, Virgen Purísima y Santa sin Pecado Concebida. Líbrame de todos los Pecados» (Cucurto 2005: 19). L'auteur joue ici avec les contradictions de l'Église, dont le pouvoir va à l'encontre des symboles sur lesquels elle s'est bâtie. Même s'il s'agit d'un saint populaire, qui ne serait certainement pas reconnu par le Vatican, surtout qu'il s'agit d'un simple homme élu chaque année, la critique se dirige bien contre l'Église catholique. Celle-ci, importée par les Européens comme un autre moyen de colonisation, est un élément capitaliste. C'est illustré par Cucurto, qui profite de son rôle pour demander aux prostituées une partie de leurs gains, devenant ainsi un «Santo Gigoló ». Ce saint gigolo dont le corps est possédé par tous et qui est rémunéré par tous est finalement un corps qui se monnaie, un corps de plus dans l'appareil capitaliste. La critique est explicitée dans un autre passage du roman, alors que Cucurto est dans son rôle de magasinier:

¡Viva el sexo pago, coño!, que es cien veces más limpio que el sexo [...] usado [...] para reproducir la raza para que los curas puedan llenar las iglesias de 
fieles [...] ¡Sin anticoncepción no hay vida, la iglesia es un simulacro de la paz mundial, la iglesia reaccionaria, fascista y acomodaticia a los gobiernos imperantes! (Cucurto 2005: 56)

Loin des scènes de promotion du saint, on trouve une authentique description de l'amour spirituel, qui unit entre eux les pécheurs, puisque le monde dans lequel évolue Cucurto est celui du péché: «La negra [...] me agarró de la cabeza y me dio un beso con mierda [...] Fue tan amargo que lo sentí dulce, fue tan odiado que lo sentí amor. "La mierda nos une, Cucu. Es la materia de los pecadores" (Cucurto 2005: 26). Cette communion, totalement antagonique à la communion catholique, est le symbole d'une opposition au monde capitaliste dans lequel s'inscrit l'Église et que Cucurto refuse, sans pouvoir pour autant s'en extraire. On trouve la même dichotomie dans le disque du groupe Bersuit Vergarabat, Hijos del culo, ceux qui souffrent la "vida boba», "los despedidos del banquete, los expulsados del paraíso ${ }^{7}$. On retrouve de nombreuses thématiques de Las aventuras del Sr. Maíz dans ce disque, notamment dans El viejo de arriba où on peut entendre: «esta noche ire hasta el fin / con los locos, los borrachos / con las putas y los guachos ... / Al zaguán de un mundo liberado/ al placer de un mambo marginal» Le seuil du monde libéré qu'évoque cette chanson sur un rythme de cachaca, un genre de cumbia, musique typiquement latinoaméricaine, très prisée par Cucurto, correspond au monde des Noirs qu'on découvre dans Las aventuras. La nuit et ses créatures - fous, prostituées et alcooliques - conduisent en marge du monde libéraliste, là où on peut se défaire des contraintes du corps social; plaisirs exutoires qui permettent aux travailleurs de s'évader d'un monde qui les tortionne.

Le yoti, c'est-à-dire le conventillo (maison à plusieurs niveaux), où vivent les ouvriers immigrés du roman, est le contre-lieu capitaliste: il est le pendant antagonique du supermarché. L'adresse en est donnée dans le roman et il se trouve au centre de Buenos Aires. Cette capitale, comme la plupart des villes américaines, partage son centre entre les activités administratives et des immeubles d'habitation pauvres. Les quartiers périphériques résidentiels aisés se trouvent autour de ce centre, cernés à leur tour par les villas miseria, les bidonvilles. Les habitants du yoti sont donc en marge de ce "monde libéré», tout en étant en son centre. On peut faire une analogie avec l'ouvrier, travaillant au cœur du système capitaliste. Les immigrés du yoti se trouvent donc doublement au cœur du néolibéralisme, tout en en étant les

7 Bersuit Vergarabat, Hijos del culo, 2000, Universal Music Argentina. El viejo de arriba, paroles et musique de Juan Subira. 
affranchis. Le yoti est décrit comme un lieu de luxure: «el lugar hereje por supremacía, el baile del sexo, la seducción y la política (Cucurto 2005: 64) .Le yoti est un trou noir dans le système rodé du capitalisme, où on puise de nouveaux travailleurs et où toutes les pulsions peuvent être assouvies. Le dieu qu'ils y choisissent est un dieu hérétique qui indique une suprématie du corps sur le spirituel chez le peuple qui l'adore. On retrouve ici le pouvoir du Noir, du sauvage, du corps du désir. Ce corps est en totale opposition avec le corps de la loi, mais aussi avec le corps du colonisé marqué par «le discours du pouvoir» dont parle Michel de Certeau dans L'écriture de l'histoire ${ }^{8}$. Le corps noir de Las aventuras paraît être vierge de cette marque. Jorgelina, une amie de Cucurto, demande à ce dernier les pages du roman qu'il lit pour s'en faire une protection menstruelle, ce que célèbre Cucurto : "qué destino terminar en la concha de una dominicana!» (Cucurto 2005: 62). Le corps des Noirs prend le dessus; l'écriture ne devient pas sens en eux, mais elle disparaît en eux, au sein même du corps. Dans le roman, cette ignorance se fait le signe de leur immunité aux lois, c'est-à-dire à l'organisation du corps social qui leur a été imposé, mais également de leur indépendance. Leur identité n'est pas annexée par l'Europe. Étant les oubliés de l'Argentine et du continent, ils sont indemnes de cette colonisation, tout en étant métissés. Ils sont les Américains. Il en va de l'emploi de la langue espagnole comme des Américains. On peut deviner dans cette déformation du castillan une identité, hautement revendiquée par le narrateur:

Salía del Supermercado en donde trabajaba reponiendo, ba, ba, bolcando papas, así con blarga [...] pero qué carajo sabe la real academia española de papas y cebollas para andar imponiendo leyes gramaticales como si fuesen líneas de cosméticos o fluidos de la atmósfera. [...] A cada estrella su pico, güey, pos para algo estamos acá, pa poner las cosas en orden, pa poner las cosas en su punto justo como hace 500 años no lo hace nadies; para implementar nuestra justicia, para meterles sus leyes gramaticales en la lanza del desgarbado Quijote de la Mancha, que acá todos somo sanchos ignorantes. ¡Y vuélvanse pa Castilla que ya se los grité en Cosa de Negros: nuestro castellano es más de Castelar que de Castilla! (Cucurto 2005: 61)

8 Michel de Certeau décrit l'arrivée d'Amerigo Vespucci en Amérique en ces termes: "Après un moment de stupeur sur ce seuil marqué d'une colonnade d'arbres, le conquérant va écrire le corps de l'autre et y tracer sa propre histoire. Il va en faire le corps historié - le blason - de ses travaux et de ses fantasmes. Ce sera l'Amérique "latine". Cette image érotique et guerrière a valeur quasi mythique. Elle représente le commencement d'un nouveau fonctionnement occidental de l'écriture. [...] Mais ce qui s'amorce ainsi, c'est une colonisation du corps par le discours du pouvoir. C'est l'écriture conquérante» (De Certeau 1975: 9). 
La langue actuelle d'Argentine et des autres pays hispanophones découle de l'expérience, qui en a modifié la grammaire, et révèle une identité distincte de l'identité européenne. Cette langue un peu différente de la langue d'origine, à peine déplacée, est incarnée par une nouvelle identité. Ce sont les corps auxquels on voulait l'imposer qui l'ont déformée.

On peut donc lire dans Las aventuras del Sr. Maíz, el héroe atrapado entre dos mundos la description de deux mondes en totale opposition. Le premier est un monde capitaliste, qui donne forme au corps social et dont le narrateur voit les sources dans la colonisation, puisque tout ce qui est capitaliste dans le roman est associé à l'Europe. C'est une colonisation qui perdure, parce que l'histoire argentine est inscrite dans l'histoire européenne, et que la conduction économique du pays dépend des intérêts internationaux. Le second est celui des Noirs, population locale, exploitée, dont les corps sont vendus et déformés par le travail, mais qui reste sourde à l'écriture des lois, peut-être précisément parce qu'elle est dénuée de droits. Le corps du héros Cucurto, attrapé entre ces deux mondes, les représente. Son corps littéraire (corps de narrateur, porteur du récit, et corps de personnage, motif du récit) est tous les corps. Il est le Noir et le capitaliste - le corps ouvrier et le corps commerçant du saint imposteur, le Noir du yoti et le magasinier du supermarché. Pris entre ces deux mondes, qu'il ne peut pas modifier, et choqué par les injustices qu'il y voit, il trouve un moyen d'évasion: le rêve. C'est grâce au rêve qu'il s'évade de son corps, représentation du corps social. Il part dans un pays libre, imaginé par lui seul: «El país de mis sueños es una mezcla del Paraguay y la República Dominicana en color Dorado. Este país no existe o sólo existe en mis sueños. Mejor, así [...] no tiene que sufrir los desplantes de la economía mundial o la globalización» (Cucurto 2005: 39).

La poésie qu'écrit le personnage Cucurto est une extension de ce rêve, toujours hors du corps. Il y clame son usage particulier de la langue écrite, qu'il «crie» (je rappelle: «ya se los grité en Cosa de Negros»). De sa poésie surgit non pas son originalité, mais son individualité. Cucurto es un poète autodidacte, qui a connu la poésie grâce à un traducteur. Il commence à écrire en copiant les poèmes qu'il préfère, les transformant ainsi à sa manière. C'est la clé de l'individualité, qui semble être dans ce roman l'unique façon de se sauver du corps social: on ne peut guère en modifier l'ordre, mais on peut le vivre à sa façon. Les lecteurs de classe moyenne qui interviennent dans le récit reprochent à Cucurto son immobilisme:

- Usted no es más que una víctima del capitalismo infame que estamos viviendo... 
- Por eso, lo invitamos a firmar este papel para exigir un plebiscito para anular los supermercados en el país.

Cucurto leur répond:

-Ustedes quieren eliminar los supermercados con sus ideas progresistas y no pueden ver la belleza de una góndola de verduras. [...] No pueden ver que en esta góndola está la vida, la selva. Miren esas arrugas en los apios, son las garras de un tigre, y esas manchitas rojas en las lechugas son los llantos de las águilas [...] Cuando nadie la oía, la góndola gruñó como un tigre, y el avispazo de un vuelo de águila se oyó al fondo, a lo lejos. (Cucurto 2005: 32)

Selon le narrateur, il ne faut pas supprimer les éléments du réel, mais les voir autrement; il ne faut pas les combattre sur le plan du réel, mais les transposer. Le poète s'oppose au corps social; la poésie est l'unique en chacun, qui sauve chaque corps. Cucurto n'est plus magasinier dans un supermarché, mais dompteur d'une nature sauvage. Copier et transformer, déplacer le sens devient une issue. $\mathrm{Si}$, comme c'est suggéré ici, le corps social est écrit par l'Europe, alors la vie individuelle réside dans l'identité propre. Le narrateur profite de la confrontation qu'il crée avec ses lecteurs pour leur dire: « ¡Todo es personal, imbéciles! Lo único que importa es la persona. ¡La 1ra. Persona!» (Cucurto 2005: 31-32) Le sujet devient un individu hors du corps, et il n'est plus un corps parmi les corps. Peut-être l'auteur cherche$\mathrm{t}$-il à nous transmettre cet apprentissage dans ce lieu de fiction qui nous comprend: «Uno no puede saberlo todo. Pero sí imaginárselo todo. Por eso estamos acá» (Cucurto 2005: 60). C'est cette imagination qui transforme le vécu et permet de se l'approprier. Cette imagination fait du conventillo un lieu de fête, accueillant un saint imposteur, avec qui tout le monde a des rapports sexuels. Transposition exubérante, parfois obscène du réel dans le realismo atolondrado propre à l'auteur, il s'agit d'un réalisme qui apporte un sens supplémentaire au réel, qui l'améliore. C'est une obscénité qui est associée à la vie, à l'affirmation d'un corps qui, s'il se monnaye et doit être déformé par le travail, est toujours vivant, animé par l'identité qui l'habite, prêt à procurer des jouissances. Cette obscénité est loin cependant d'une autre forme d'impudeur qu'aurait été celle des descriptions de corps meurtris, broyés, tués. La torture n'est plus aussi courante en Argentine en 1990 qu'en 1970, mais la population dont parle l'auteur est victime d'interrogatoires musclés, assassinats, viols, malnutrition, ou manque de soins médicaux. Dans ce roman, il n'y a pas de mort, que de la vie. La maltraitance et l'exploitation sont suggérées, il serait aussi difficile de les oublier que la présence des «Blancs» qui cernent le récit. L’auteur a choisi une autre réalité, sur un mode hurlant et coloré, parfois brutal, mais dans 
le registre de la vie. Il choisit ici de révéler une identité en évolution. La langue espagnole est maltraitée, on l'a vu, parce qu'elle est envahie par cette identité. Les sonorités très travaillées de Las aventuras en font un texte oral, qui refuse là encore les lois de la langue écrite et, à travers elle, les lois du colonisateur. Le lecteur reçoit une chanson rythmée et unique, marquée par une identité. Cette dernière n'est pas exclusive, l'auteur s'inspire de tous les argots qu'il entend; une langue métissée donc, mais surtout une langue avec laquelle il joue, ce qui est peu courant dans la littérature argentine. C'est le signe d'une littérature en constante transformation, littérature vivante, qui cherche de nouvelles personnes d'énonciation, de nouvelles frontières avec le réel, des narrateurs et des narrataires qui se mêlent; des auteurs et des personnages qui partagent leur nom. Cette matière labile est le signe d'une identité à construire, qui n'est pas et ne sera jamais définitive. C'est aussi évidemment une écriture politique, la satire du capitalisme l'indique assez. Mais, on l'a vu, c'est une critique drôle, haute en couleurs, peut-être plus dangereuse qu'une autre forme de menace, dans la mesure où elle nie le pouvoir, comme l'est le recueil de poèmes Zelarayán dont le narrateur nous raconte l'écriture:

Escribo. Comienza mi venganza, más letal que el mayor impuesto que pueda tener nunca el supermercadismo argentino. Pelo mi libro Zelarayán. ¡Cómo me divierto escribiéndolo! Es una burla a la clase media argentina y a sus modos, gustos y costumbres. Ataco y destruyo la buena literatura sin piedad. Juan L. Ortiz, Lamborghini, Copi, Zelarayán, Zurita, Millán, Elvira Hernández, Cisneros, Hinostroza, Maquieira, Desiderio, Edwards, Vallejo, Gelman, Gonzalo Rojas. Antes todos se reían de mí, ahora es mi risa la que asoma en el mundo, de entre las góndolas de un supermercado para vengarse de todo, mi horrible risa que es lo único que tengo y ahora soy el que se ríe de todos sin parar.

De sujet, Cucurto est devenu à ce moment-là individu. Mais ce baptême n'est pas irréversible et la fiction est en perpétuel mouvement. Aussi ajoutet-il: «Años después, pienso que en esa lista también entraría yo y no veo la hora de destruirme a mí mismo. Quizá ya lo estoy haciendo. Dios quiera» (Cucurto 2005: 40-41). 


\section{Bibliographie}

Cucurto, 2005, Las aventuras del Sr. Maíz, el héroe atrapado entre dos mundos, Buenos Aires, Interzona latinoamericana.

De Certeau, Michel, 1975, L'écriture de l'histoire, Paris, Éditions Gallimard, Folio histoire.

Fuentes, Carlos, 1990, Todos los gatos son pardos, México, siglo XXI.

Nicolini, Fernanda, 2006, «De marginal a escritor de culto», Noticias, $\mathrm{n}^{\circ} 1566$, décembre 2006, publication virtuelle:

http://www.revista-noticias.com.ar/comun/nota.php?art=137\&ed=1566

Noé, Luis Felipe, 2003, Una sociedad colonial avanzada, 1971-2003, Buenos Aires, Asunto impreso. 